\title{
The Impact of Stock Market Structure on Volatility: Evidence from a Call Auction Suspension
}

\author{
Silvio John Camilleri ${ }^{1}$ \\ ${ }^{1}$ Banking and Finance Department, Faculty of Economics, Management \& Accountancy, University of Malta, Msida, \\ Malta
}

Correspondence: Silvio John Camilleri, Banking and Finance Department, Faculty of Economics, Management \& Accountancy, University of Malta, Msida MSD 2080, Malta. E-mail: silvio.j.camilleri@um.edu.mt

Received: February 26, 2015

Accepted: March 16, 2015

Online Published: March 23, 2015

doi:10.5430/ijfr.v6n2p44

URL: http://dx.doi.org/10.5430/ijfr.v6n2p44

\begin{abstract}
The purpose of this paper is to investigate the volatility impacts of the suspension of a call auction system by the National Stock Exchange of India (NSE) in June 1999, thus extending prior empirical work relating to this area. The realised volatility on NSE is compared with that of the Bombay Stock Exchange using two volatility proxies: modulus of $\log$ returns and scaled intra-day price difference. We also focus on conditional volatility by estimating an AGARCH model on seasonally-adjusted NSE Nifty Index data. Whilst some results yield contrasting inferences, the overall outcomes indicate that volatility was higher during the auction period, and we do not find any evidence that supports the foreseen benefits of auction frameworks. Results reinforce the idea that market designers should think about the possible interactions with subsidiary market microstructure features when formulating auction protocols, since the latter may compromise auction efficacy.
\end{abstract}

Keywords: call auctions, National Stock Exchange of India, seasonality, stock markets, volatility

\section{Introduction}

The evaluation of different trading protocols is one of the salient branches of market microstructure studies, given its relevance to traders and market designers. The way in which securities markets' structure can affect the pricing process, volatility, liquidity and trading costs, took a prominent role on the research agenda since the study of Demsetz (1968), which suggested that factors such as the number of traders can affect the spread. The efficacy of call auctions, as compared to other market frameworks such as continuous trading takes a prominent role in this strand of literature. Major trading venues such as the New York Stock Exchange, NASDAQ, the London Stock Exchange, and Xetra use call auctions to open or close their trading sessions.

In a call auction framework, incoming orders are batched together and executed simultaneously at a common price. Therefore call auctions are expected to aggregate information more efficiently, since a wider cross-section of orders are taken into consideration when determining prices (Economides and Schwartz, 1995). This contrasts with continuous trading, where transactions take place at any instant when two orders on the opposite side of the market may be matched. In such frameworks prices are established during the course of trading, implying that transactions may occur at 'false' prices until the fundamental value of the security is discovered (Schwartz, 2000). If auctions accelerate price discovery, one may expect a lower degree of price dispersion around fundamental values. Despite this, such assertion is not fully supported by empirical literature which offers contradictory overall evidence.

In this paper we evaluate the effectiveness of call auctions in restraining volatility, by focusing on the impact of the suspension of opening and closing auctions by the National Stock Exchange of India (NSE) on $9^{\text {th }}$ June 1999 . This occurrence was considered in prior studies by Camilleri and Green (2009) and Camilleri (2015), which offer mixed evidence as to the overall efficacy of these auctions. In the former investigation it was found that intra-day volatility decreased significantly following the suspension, yet overnight return reversals became significantly higher. Camilleri (2015) reported that the auction suspension was followed by lower return volatility during the middle and the closing parts of the trading day, and higher volatility in the opening returns.

We believe that there is still further potential for analysing the volatility changes around this auction suspension. In particular we compare the realised volatility on NSE with the volatility on the Bombay Stock Exchange (BSE) to 
inquire whether the auction resulted in lower volatility on NSE in relative terms. In addition, we consider changes in longer term conditional volatility on NSE through the estimation of an Absolute Value GARCH (AGARCH) model (Heutschel, 1991). Longer term volatility was sidelined in the former papers on the grounds that market microstructure changes mainly affect stock prices in the short term. Yet, one may deduce that reductions in short term intra-day volatility may also result in lower longer term volatility. In particular, if closing auctions were contributing to a more efficient market price, this should be reflected through lower dispersion in the time series of closing prices around the fundamental value of the index portfolio.

The contributions of this paper are twofold. Firstly, the auction suspension we are analysing occurred in the context of a system which involved no other significant market microstructure changes during the particular period. This is a noteworthy aspect since modifications in trading protocols typically involve several simultaneous reforms which make it unclear whether any impacts are due to a given particular factor (Amihud, Mendelson and Lauterbach, 1997). Secondly we focus on both realised and modelled volatility, and one may expect to capture a more thorough comprehension of the issue at hand when considering both types of volatility (Zhang and $\mathrm{Hu}, 2013$ ).

The paper is structured as follows: we review the relevant literature in section 2, whilst section 3 offers information about the empirical setting and the data set. In section 4, we compare the relative volatilities on NSE and BSE, and in section 5 we investigate the impact of the auction suspension by estimating an AGARCH model on daily data. A summary of the main findings and insights is shown in section 6 .

\section{Literature Review}

Trading frameworks may either rely exclusively on call auctions or continuous trading, or else the protocol may provide for a hybrid system. Call auctions differ in their structure and such variations may have distinct implications on the trading process (Comerton-Forde and Rydge, 2006). In addition, the market context in which auctions are held may also affect their efficacy and this partly accounts for the mixed evidence outlined below.

One determining factor which impacts on auction efficacy is the information signals sent by traders when submitting orders. In the theoretical study of Madhavan (1992) and in the analysis of Economides and Schwartz (1995) it was noted that call auctions aggregate the expectations of a cross section of traders and thus augment price discovery. One may deduce that this feature contributes towards a lower dispersion of prices around fundamental values, and therefore a lower overall volatility. Contradictory insights from the theoretical model of Caillaud and Mezzetti (2004) suggest that traders may use the auction framework strategically so that they hold back information to trade upon it profitably at a subsequent stage.

The theoretical study of Chakraborty, Pagano and Schwartz (2012) and the experimental study of Biais, Bisière and Pouget (2014) suggest that the information dissemination benefits of auctions may be enhanced through a pre-opening session, where market participants can submit non-binding orders, thereby exchanging opinions about the fundamental values of stocks. Chakraborty, Pagano and Schwartz (2012) suggested that a trading venue may offer both a transparent pre-open process and an additional hidden facility for those traders who would like to conceal their orders. The latter feature could be used as a stock of orders for the purpose of reducing imbalances on either side of the market, rather than to establish an opening price.

Biais, Hillion and Spatt (1999) noted that on the (former) Paris Bourse, the orders posted during the initial phases of the pre-opening auction may be classified as 'noise' and price discovery mainly occurs at the final phase of the auction. Conversely, studies focusing on the Toronto Stock Exchange (Davies, 2003) and the West-African Bourse (Dia and Pouget, 2011) suggested that the majority of pre-opening orders were submitted with an actual purpose to trade.

Prior literature has also focused on the idea that call auctions may be used to misguide other traders, for instance through the submission of mispriced orders which are subsequently withdrawn. Comerton-Forde and Rydge (2006) studied how diverse auction algorithms handle manipulative orders. When considering a less liquid stock, the auction established a price in the presence of a manipulative order, yet no price was set when such order was eliminated. This challenges the traditional thought that auctions facilitate the trading of less liquid stocks, by creating a deeper market through aggregating orders. Pinfold and He (2012) analysed the implementation of a closing call auction on the New Zealand securities market and noted lower potential for market manipulation and increased pricing efficiency in the auction setup.

In order to minimise the possibility that traders misguide the markets through manipulative orders, the auction may include features such as a random market opening time or the requirement that orders submitted during the auction remain binding up to the termination of the auction (Biais, Bisière and Pouget, 2014). 
Overall, this strand of literature suggests that auctions may process information more efficiently; however potential manipulators may take advantage of this feature to misguide other participants. Given the diverse implications which such trends may have on pricing efficiency and volatility, one may not form straightforward expectations regarding the potential outcomes of the auction suspension which we are considering in this paper.

The efficacy of auctions may be compromised by lack of trading interest. Madhavan and Panchapagesan (2000) found that in case of the New York Stock Exchange opening auction, less liquid stocks were more prone to mispricing owing to order imbalances. Chakraborty, Pagano and Schwartz (2012) proposed that trading venues may introduce an animator to take the opposite side of orders and address such imbalances.

The notion that lack of trading activity may lessen auction efficacy should not be sidelined in the context of the NSE auction suspension which we tackle in this study. For instance, Camilleri and Green (2009) reported that only 7\% to $18.6 \%$ of the less liquid stocks in their sample traded in the NSE opening auction. Nonetheless, given that in this paper we use index data which are computed through a portfolio of highly liquid stocks, such factors might not have any direct impacts on our findings.

Various empirical papers have considered the volatility impacts of the introduction of auctions in a trading venue, or opted for comparative studies across exchanges. For instance, Ronen (1998) studied the impact of the change from a closing call auction to an opening call auction system on the Tel-Aviv Stock Exchange in 1988. The author reported that this modification did not result in material changes in volatility during the initial stages of the trading session.

Chang et. al. (2008) analysed the implementation of call auctions at the start and at the end of the trading day on the Singapore Stock Exchange which took place in the year 2000. It was noted that this initiative curtailed volatility especially in the case of liquid stocks. The latter notion was not fully confirmed by Chelley-Steeley (2008) who investigated the inclusion of a closing call auction on the London Stock Exchange in 1997. The author found that the resulting increase in pricing efficiency was more pronounced in the case of less liquid stocks.

Comerton-Forde (1999) investigated stock return data from the Australian Stock Exchange and the Jakarta Stock Exchange. The former exchange commences with a call auction, while the latter one commences with continuous trading. The author concluded that auctions reduce volatility at the initial phases of the trading sessions.

Kandel, Rindi and Bosetti (2012) analysed the impacts of closing auctions on the Borsa Italiana and the (former) Paris Bourse. They concluded that these auctions curtailed volatility, lowered the spreads and reduced trading volumes during the last minutes of the continuous trading session. This was attributed to market participants becoming less impatient to trade during this period due to the availability of the auction. The spread reductions materialised in lower volatility due to a diminished bid-ask bounce effect.

Pagano, Peng and Schwartz (2013) considered the introduction of opening and closing call auctions on NASDAQ in 2004 and reported an impact on the continuous trading session in terms of a drop in volatility especially towards the closing. Aitken, Comerton-Forde and Frino (2005) analysed the introduction of closing call auctions on the Australian Stock Exchange in 1997, and noted that on high-volatility days there was a pronounced tendency for traders to postpone their trading towards the auction. They suggested that call auctions prove more useful to traders on high-volatility days.

Overall, the prior studies which considered the introduction of call auctions seem to point at reduced volatility following the implementation of these trading frameworks. Thus, one may expect that an auction suspension as considered in this paper may be followed by increased volatility. A different outcome could constitute an interesting addition to the literature which may call for the re-thinking of the former findings.

Trading venues also use auctions in order to stabilise the market following unusually large price movements such as a flash-crash. Brewer, Cvitanic and Plott (2013) set up experimental market microstructure settings and found that auctions were the most suited framework to stabilise prices following such occurrences. This corroborates the findings of Reboredo (2012) who reported that on the Spanish Stock Exchange, call auctions held following abnormal price movements contributed towards lower volatility.

Prior studies have also tackled the empirical case which we are considering in this paper. Camilleri and Green (2009) reported contradictory evidence regarding volatility changes following the auction suspension on NSE. They noted a significant reduction in price dispersion during the trading day but a significant increase in volatility in terms of the tendency for overnight returns to reverse at a subsequent stage. Camilleri (2015) found an overall reduction in volatility coefficients, when fitting GARCH models on intra-day data. The author also reported an increased variance in opening return distributions sampled at one-minute intervals, but reduced variances in return distributions for the remaining part of the trading day. 
It is the aim of this paper to glean further empirical evidence so that the impacts of this auction suspension may be assessed more clearly by using different methodologies and through the comparison of NSE and BSE data. Given that the theoretical relationship between call auctions and volatility is still unresolved due to contrasting findings, we believe that this empirical study offers a contribution towards addressing this gap in the literature.

\section{Empirical Context and Data}

The NSE and the BSE are the main stock exchanges in India and these venues compete for listings and order flow. Major Indian stocks are quoted on both venues. The exchanges are characterised by a broad range of stocks and high trading volumes in the case of liquid securities.

The setting up of NSE in 1994 resulted in considerable restructuring of the Indian market through the promotion of transparency and better organised frameworks for settlement, securities lending and derivatives trading. The number of equities trading on NSE as at November 2014 stood at approximately 1,600, whilst the volume of a typical trading day on the exchange was around 7 million transactions.

In the late 1990s, NSE introduced opening and closing call auctions to complement its order-driven system. During the intermediate part of the day, trading occurred through a continuous limit order book. The auctions established prices with the main aim of maximising trading volume. During the auction, orders could be modified or cancelled, while orders which included bargain conditions such as 'all-or-none' were not taken into consideration.

The auctions were suspended on 9th June 1999, probably due to software-related problems. Camilleri and Green (2009) established that the software glitches did not affect the pricing process, with the possible exception of the closing auction held on the 8th June. The authors also noted that closing auctions were more active than the opening ones.

The main data set used in this investigation consists of daily observations of the NSE Nifty Index. This is one of the principal indices published by NSE which includes 50 large-capitalisation securities. The observations used for assessing volatility changes following the auction suspension, run from 1st February 1999 to 16th November 1999. When comparing NSE volatility with that prevailing on BSE, we also use the data for the S\&P BSE 100 Index which includes the 100 largest companies traded on BSE. When adjusting the original data for seasonality as required in section 5, we use a longer time series of the Nifty index observations to glean information about seasonality patterns - from January 1997 to December 2001.

\section{Comparing NSE and BSE Volatility}

In order to assess the efficacy of the NSE auctions in curtailing volatility, we first compared the volatility of the Nifty Index with that of the S\&P BSE 100, using the data sample from 1st February to 16th November 1999. (Note 1) Since both indices comprise large-capitalisation Indian firms and given that a number of issues trade on both exchanges, it is likely that any relative changes in volatility emanate from market microstructure features, such as whether call auctions were held at specific stages of the trading day.

We used two volatility proxies in this comparison: the modulus of log returns, and the scaled intra-day price difference which is equal to the difference between the highest and the lowest index value during the trading day, divided by the opening value.

We thus estimated the following two models:

$$
\begin{gathered}
\left|y_{t}\right|=\pi+\alpha\left|y_{t}^{b}\right|+\beta d_{a, t}+\varepsilon_{t} \\
s_{t}=\pi+\alpha s_{t}^{b}+\beta d_{a, t}+\varepsilon_{t}
\end{gathered}
$$

where $y t$ denotes the log return on NSE on day $t, y^{b}{ }_{t}$ is the log return on BSE on day $t, s_{t}$ and $s_{t}{ }_{t}$ are the scaled intra-day price difference on day $t$ on NSE and BSE respectively, $d_{a, t}$ is a dummy variable which took a value of one during the auction period and zero thereafter, $\pi, \alpha$, and $\beta$ are parameters and $\varepsilon_{t}$ is an error term. If the volatility on NSE was comparatively lower during the auction period, one would expect a negative $\beta$ in both models.

Results shown in Table 1 indicate that $\beta$ is not statistically significant, and it took a positive value in the first model and a negative value in the second one. Given this, these results did not yield a clear insight regarding the volatility impact of the auction suspension. 
Table 1. NSE and BSE volatility comparisons

\begin{tabular}{|c|c|c|c|}
\hline \multicolumn{4}{|c|}{ PANEL A: Modulus of Closing Returns as Volatility Proxy } \\
\hline & $\pi$ (intercept) & $\alpha\left(\right.$ coefficient of $\left.\left|y_{t}^{b}\right|\right)$ & $\beta\left(\right.$ coefficient of $\left.d_{a, t}\right)$ \\
\hline \multirow[b]{3}{*}{$R^{2}$} & 0.0008 & \multirow{2}{*}{$\begin{array}{c}0.8858 * * * \\
(22.49)\end{array}$} & 0.0011 \\
\hline & $(0.96)$ & & $(1.09)$ \\
\hline & 0.7303 & Adjusted $R^{2}: \quad 0.7275$ & Number of Observations: 197 \\
\hline \multicolumn{4}{|c|}{ PANEL B: Scaled Intra-Day Price Difference as Volatility Proxy } \\
\hline & $\pi$ (intercept) & $\alpha\left(\right.$ coefficient of $\left.s_{t}^{b}\right)$ & $\beta\left(\right.$ coefficient of $\left.d_{a, t}\right)$ \\
\hline & $0.0050 * * *$ & $0.8642 * * *$ & -0.0005 \\
\hline & $(5.40)$ & $(25.21)$ & $(0.54)$ \\
\hline$R^{2}$ & 0.7681 & Adjusted $R^{2}: 0.7658$ & Number of Observations: 198 \\
\hline
\end{tabular}

Description: The table shows the results obtained when regressing NSE volatility proxies, over the corresponding ones for BSE and a dummy variable $d_{a, t}$ which took a value of one during the call auction period and zero otherwise. Panel A shows the estimation using the modulus of closing log returns $\left(\left|y_{t}\right|\right.$ and $\left.\left|y_{t}^{b}\right|\right)$, whilst Panel B shows the estimation using the scaled intra-day price difference $\left(s_{t}\right.$ and $\left.s_{t}^{b}\right)$ as volatility proxies. Regression coefficients are shown on top and t-ratios are reported in brackets underneath. Significance at the $99 \%$ level of confidence is denoted by ***.

\section{Modelling Volatility Changes through Absolute Value GARCH}

One major challenge encountered in the investigation of longer term volatility on NSE is the monthly seasonality which includes an increase in volatility following the closing of the accounting years of many Indian companies in March. Whilst seasonality was not problematic in the previous models (since it was common to both data sets which were compared), it made the NSE data biased against the auction regime for the purposes of the subsequent investigation. The higher volatility month coincided with the call auction period while other low volatility months fell within the auction suspension period. In this way, we adjusted the data for seasonality given that this is not related to the auctions.

We thus extended the original data set, in order to glean information about monthly seasonality on NSE and make the required adjustments. For this purpose we used a time series of the Nifty closing values from 2nd January 1997 to 31st December 2001. We estimated an OLS regression with the log return modulus as the dependant variable, whilst the explanatory variables were eleven dummies for the months of February to December. Results confirmed a significant increase in volatility during April and a significant reduction in volatility during August, November and December (Table 2 Panel A).

In order to use a parsimonious model, a further regression was estimated as follows:

$$
\left|y_{t}\right|=\pi+\alpha d_{a p r, t}+\beta d_{\text {aug }, t}+\chi d_{n o v, t}+\delta d_{d e c, t}+\varepsilon_{t}
$$

where $y t$ denotes the log return on NSE on day $t, d_{a p r, t}, d_{\text {aug }, t}, d_{n o v, t}$, and $d_{d e c, t}$ are four dummy variables for the months of April, August, November and December which took a value one during the respective month and zero otherwise, $\pi, \alpha, \beta, \chi$, and $\delta$ are parameters and $\varepsilon_{t}$ is an error term. Results shown in Table 2 Panel B confirm the underlying seasonality pattern.

In order to adjust for the observed seasonality, we calculated the overall average of log return moduli for the five-year time series, and then estimated the averages for each of the five sub-sets consisting of April, August, November, December and rest-of-the-year observations. For each sub-set, we then computed the ratio of the sub-set average to the overall average. These ratios were then used as divisors on the original observations, in order to scale 
the data for each sub-set. In this way we obtained a more uniform data dispersion, across the respective periods of the year.

Table 2. Monthly seasonality of volatility

Panel A:

Estimation using Eleven Dummy Variables

\begin{tabular}{|c|c|c|c|c|c|}
\hline \multirow[b]{2}{*}{ Intercept } & Coefficient & (T-ratio) & & Coefficient & (T-ratio) \\
\hline & $0.0145 * * *$ & $(12.01)$ & $\pi($ Intercept $)$ & $0.0136 * * *$ & $(32.25)$ \\
\hline \multicolumn{3}{|c|}{ Dummy Variables: } & \multicolumn{3}{|l|}{ Dummy Variables: } \\
\hline February & -0.0023 & $(1.33)$ & & & \\
\hline March & 0.0023 & $(1.39)$ & $\alpha\left(\right.$ coeff. of $\left.d_{a p r, t}\right)$ & $0.0047 * * *$ & $(3.59)$ \\
\hline April & $0.0039 * *$ & $(2.24)$ & & & \\
\hline May & -0.0011 & $(0.67)$ & $\beta$ (coeff. of $d_{\text {aug }, t}$ ) & $-0.0034 * * *$ & $(2.65)$ \\
\hline June & -0.0012 & $(0.71)$ & & & \\
\hline July & -0.0027 & $(1.64)$ & $\chi\left(\right.$ coeff. of $\left.d_{n o v, t}\right)$ & $-0.0025 * *$ & $(1.97)$ \\
\hline August & $-0.0042 * *$ & $(2.48)$ & & & \\
\hline September & -0.0021 & $(1.23)$ & $\delta$ (coeff. of $\left.d_{d e c, t}\right)$ & $-0.0028 * *$ & $(2.23)$ \\
\hline October & 0.0004 & $(0.26)$ & & & \\
\hline November & $-0.0034 * *$ & $(1.98)$ & & & \\
\hline December & $-0.0037 * *$ & $(2.16)$ & & & \\
\hline \multicolumn{2}{|l|}{$\mathrm{R}^{2}$} & 0.0356 & \multicolumn{2}{|l|}{$\mathrm{R}^{2}$} & 0.0246 \\
\hline \multicolumn{2}{|l|}{ Adjusted $\mathrm{R}^{2}$} & 0.0270 & \multicolumn{2}{|l|}{ Adjusted $\mathrm{R}^{2}$} & 0.0214 \\
\hline \multicolumn{2}{|l|}{ F-statistic } & 4.1298 & \multicolumn{2}{|l|}{ F-Statistic } & 7.7960 \\
\hline \multicolumn{2}{|c|}{ Number of Observations } & 1244 & \multicolumn{2}{|c|}{ Number of Observations } & 1244 \\
\hline
\end{tabular}

Panel B:

Parsimonious Model .

Decsription: In the above models, volatility was measured in terms of the log return moduli. In the model shown in Panel A, the explanatory variables constituted of an intercept and eleven dummy variables for the months of February to December. In the parsimonious model shown in Panel B, the explanatory variables constituted of an intercept and four dummy variables for the months of April, August, November and December. Statistical significance is denoted by $* * *$ and $* *$ for the $99 \%$ and $95 \%$ confidence levels respectively.

We then used the seasonally adjusted data from 1st February to 16th November 1999 in order to assess volatility changes following the auction suspension. As a preliminary test, we regressed the modulus of seasonally-adjusted Nifty returns as follows:

$$
\left|y_{t}^{*}\right|=\pi+\beta d_{a, t}+\varepsilon_{t}
$$

where $y^{*}$ is the seasonally-adjusted $\log$ return on day $t, d_{a, t}$ is a dummy variable which took the value of one during the call auction period and zero thereafter, $\pi$ and $\beta$ are estimated coefficients, and $\varepsilon_{t}$ is an error term. The results shown in Table 3 indicate that volatility was higher during the call auction period, however the auction dummy is not statistically significant.

We then progressed with the estimation of an AGARCH model using the seasonally-adjusted Nifty returns. In order to select the model which best captures the return generating process, we estimated six $\operatorname{AR}(\rho)$ models, with $\rho$ ranging from 0 to 5. Both the Schwarz Bayesian Criterion and the Akaike Information Criterion selected an AR(0) process. LM heteroskedasticity tests (Engle; 1982) rejected the null hypothesis of no ARCH effects at the $95 \%$ level of confidence. (Note 2) 
Table 3. Regressing seasonally-adjusted log return moduli on a call auction dummy

\begin{tabular}{cccc}
\hline $\boldsymbol{\pi}$ (Intercept) & $\boldsymbol{\beta}$ (coefficient of $\left.\boldsymbol{d}_{\boldsymbol{a}, \boldsymbol{t}}\right)$ & \multicolumn{2}{c}{ Explanatory Statistics } \\
$0.0129 * * *$ & 0.0018 & $\mathrm{R}^{2}$ & 0.0054 \\
$(11.62)$ & $(1.05)$ & ${\text { Adjusted } \mathrm{R}^{2}}^{2}$ & 0.0005 \\
\hline
\end{tabular}

Description: The table shows the results obtained when regressing the seasonally-adjusted Nifty log return moduli $\mid y^{*} t$, over an intercept $\pi$, and a dummy variable $d_{a, t}$ which took the value of one during the call auction period and zero otherwise. The time series comprised 204 observations. Regression coefficients are shown on top and t-ratios are reported underneath. Significance is denoted by $* * *$ for the $99 \%$ confidence level.

An AGARCH model was then estimated, where returns were modelled as an AR(0) process and conditional volatility was modelled as follows:

$$
\sqrt{h_{t}}=\pi+\alpha\left|\varepsilon_{t-1}\right|+\beta \sqrt{h_{t-1}}+\omega d_{a, t}
$$

where $h_{t}$ is the conditional standard deviation of the error term at time $t, \varepsilon_{t}$ is the unexpected return observed during day $t, d_{a, t}$ is an auction dummy as defined above and $\pi, \alpha, \beta$ and $\omega$ are coefficients.

Estimations shown in Table 4, yielded a positive (yet statistically insignificant) coefficient for the dummy variable $d_{a, t}$, indicating a higher conditional volatility during the auction regime. The results of this test are thus consistent with the previous one, in that we obtained weak evidence against the call auction setup.

Table 4. AGARCH Model with an auction dummy

\section{Return Generating Process AR(0):}

Intercept of $\mathrm{AR}(0)$ process

0.0022

\begin{tabular}{lr}
\multicolumn{2}{c}{ Explanatory Statistics } \\
$\mathrm{R}^{2}$ & -0.00003 \\
Adjusted $\mathrm{R}^{2}$ & -0.01518
\end{tabular}

Explanatory Statistics

Adjusted $\mathrm{R}^{2}$

\section{Conditional Variance Equation:}

$\begin{array}{cccc}\pi & \alpha & \beta & \omega \\ 0.0176 & 0.1603 * * & -0.1541 & 0.0015 \\ (0.13) & (1.81) & (0.19) & (0.56)\end{array}$

Wald Test Statistic for the null hypothesis that $\alpha+\beta+\omega=0$ :

0.0001 as compared to a Chi Squared (1) Critical Value of 2.71 at the $90 \%$ level of confidence.

Wald Test Statistic for the null hypothesis that $\alpha+\beta=0$ :

0.00006 as compared to a Chi Squared (1) Critical Value of 2.71 at the $90 \%$ level of confidence.

Wald Test Statistic for the null hypothesis that $\omega=0$ :

0.3161 as compared to a Chi Squared (1) Critical Value of 2.71 at the $90 \%$ level of confidence.

Description: The table shows the results for an AGARCH process estimated on the Nifty Index log returns (202 observations). The conditional volatility equation included a dummy variable $\left(d_{a, t}\right)$ which took a value of one during the call auction period, and zero otherwise. The t-ratios are shown in brackets underneath the respective coefficients. Significance at the $95 \%$ confidence level is denoted by $* *$.

\section{Conclusion}

In this paper we analysed volatility changes following the suspension of opening and closing call auctions on NSE. We compared the volatility prevailing on the NSE with that on BSE, using the log return moduli and the scaled intra-day price differences. The inclusion of an auction dummy in the respective models yielded contrasting (and statistically insignificant) indications as to whether the auction suspension resulted in higher or lower volatility on 
NSE when compared to its counterpart.

We also focused on volatility trends in NSE inter-day data, following adjustments for seasonality prevailing on the Indian markets. Tests on log return moduli revealed that realised volatility was higher during the auction period; however the difference was not statistically significant. Modelling volatility through an AGARCH process reinforced this indication, since conditional volatility was found to be higher during the auction period, although the dummy variable was insignificant.

Collectively, these outcomes may be interpreted as mild evidence against the auction setup. These findings challenge the intuition that by aggregating the orders of a cross-section of traders, call auctions enhance price discovery and reduce the dispersion of prices around fundamental values. The above evidence is in line with that of Camilleri and Green (2009) and Camilleri (2015) that the NSE call auctions were less effective than one may expect. Despite this, the results of this paper differ from prior studies outlined above which associated the presence of call auctions with reduced volatility (Comerton-Forde, 1999; Chang et. al., 2008; Pagano, Peng and Schwartz, 2013).

This suggests that the auction structure should be carefully thought out, in order to increase the likelihood that any expected benefits materialise in practice. Factors that may have compromised the NSE auction efficacy include the possibility that its setup was not suitable for the particular market characteristics or that its effectiveness was hindered by interactions with other market features. For instance, the transparency of an auction is an aspect which may prove particularly challenging. Low transparency levels might preclude correctly-priced orders from being displayed to the market when the fundamental value deviates considerably from the market clearing price. Conversely, increased transparency may facilitate collusion between traders by making it easier to infer other participants' strategies, and it also increases the cost of the "free option" problem related to limit orders (Madhavan, Porter and Weaver, 2005). In addition, the relationship between pre-trade transparency and market quality is not necessarily a linear one (Eom, Ok and Park, 2007) and it may be affected by factors such as market depth (Frutos and Manzano, 2014).

Designers should also consider whether auctions make sense in the particular circumstances; for instance the use of auctions to manipulate stock prices (Comerton-Forde and Rydge, 2006) may have been more prevalent in India where markets were traditionally prone to dubious trading practices (Shah and Sivakumar, 2000; Agarwal and Singh, 2006). Trading venues may thus implement features to reduce the potential for submitting manipulative orders, such as a random market opening time or restrictions on the cancellation of orders during the auction (Biais, Bisière and Pouget, 2014).

In addressing potential order imbalances, exchanges may consider the inclusion of an animator who takes the opposite side of orders to facilitate trading during the auction (Chakraborty, Pagano and Schwartz, 2012). These considerations suggest that further research is required to assess how the auction structure or other subsidiary market features may compromise auction effectiveness, as was apparently taking place on NSE.

\section{Acknowledgements}

The author thanks Christopher J. Green (Loughborough University) for extensive discussions about former versions of the paper. The kind comments of Steve Thomas (Cass Business School), Lawrence Leger (Loughborough University), Yusho Kagraoka (Musashi University) and participants at the Portuguese Finance Network Conference (2010) are also acknowledged.

\section{References}

Agarwal, M., \& Singh, H. (2006). Merger announcements and insider trading activity in India: An Empirical Investigation. Investment Management and Financial Innovations, 2006(3), 140-154. Retrieved from http://businessperspectives.org/journals_free/imfi/2006/imfi_en_2006_03_Agarwal.pdf

Aitken, M., Comerton-Forde. C., \& Frino, A. (2005). Closing call auctions and liquidity. Accounting and Finance, 45, 501-518. http://dx.doi.org/10.1111/j.1467-629X.2005.00155.x

Amihud, Y., Mendelson, H., \& Lauterbach, B. (1997). Market microstructure and securities values: Evidence from the Tel Aviv Stock Exchange. Journal of Financial Economics, 45(3), 365-390. http://dx.doi.org/10.1016/S0304-405X(97)00021-4

Biais, B., Bisière, C., \& Pouget, S. (2014). Equilibrium discovery and preopening mechanisms in an experimental market. Management Science, 60(3), 753-769. http://dx.doi.org/10.1287/mnsc.2013.1787

Biais, B., Hillion, P., \& Spatt, C. (1999). Price discovery and learning during the preopening period in the Paris Bourse. Journal of Political Economy, 107(6), 1218-1248. http://dx.doi.org/10.1086/250095 
Brewer, P., Cvitanic, J., \& Plott, C.R. (2013). Market microstructure design and flash crashes: a simulation approach. Journal of Applied Economics, 16(2), 223-250. http://dx.doi.org/10.1016/S1514-0326(13)60010-0

Caillaud, B., \& Mezzetti, C. (2004). Equilibrium reserve prices in sequential ascending auctions. Journal of Economic Theory, 117(1), 78-95. http://dx.doi.org/10.1016/j.jet.2003.11.002

Camilleri, S.J. (2015). Do call auctions curtail price volatility? Evidence from the National Stock Exchange of India. Managerial Finance, 41(1), 67-79. http://dx.doi.org/10.1108/MF-10-2013-0292

Camilleri, S.J., \& Green, C.J. (2009). The impact of the suspension of opening and closing call auctions: Evidence from the National Stock Exchange of India. International Journal of Banking, Accounting and Finance, 1(3), 257-284. http://dx.doi.org/10.1504/IJBAAF.2009.022716

Chakraborty, A., Pagano, M.S., \& Schwartz, R.A. (2012). Order revelation at market openings. Journal of Financial Markets, 15(2), 127-150. http://dx.doi.org/10.1016/j.finmar.2011.08.002

Chang, R.P., Ghon Ree, S., Stone, G.R., \& Tang, N. (2008). How does the call market method affect price efficiency? Evidence from the Singapore Stock Market. Journal of Banking \& Finance, 32(10), 2205-2219. http://dx.doi.org/10.1016/j.jbankfin.2007.12.036

Chelley-Steeley, P.L. (2008). Market quality changes in the London Stock Market. Journal of Banking \& Finance, 32(10), 2248-2253. http://dx.doi.org/10.1016/j.jbankfin.2007.12.049

Comerton-Forde, C. (1999). Do trading rules impact on market efficiency? A comparison of opening procedures on the Australian and Jakarta Stock Exchanges. Pacific-Basin Finance Journal, 7(5), 495-521. http://dx.doi.org/10.1016/S0927-538X(99)00020-7

Comerton-Forde, C., \& Rydge, J. (2006). Call auction algorithm design and market manipulation. Journal of Multinational Financial Management, 16(2), 184-198. http://dx.doi.org/10.1016/j.mulfin.2005.06.002

Davies, R.J. (2003). The Toronto Stock Exchange preopening session. Journal of Financial Markets, 6(4), 491-516. http://dx.doi.org/10.1016/S1386-4181(02)00018-6

Demsetz, H. (1968). The cost of transacting. Quarterly Journal of Economics, 82(1), 33-53.

Dia, M., \& Pouget, S. (2011). Sunshine trading in an African stock market. Managerial Finance, 37(3), 257-74. http://dx.doi.org/10.1108/03074351111113315

Economides, N., \& Schwartz, R.A. (1995). Electronic call market trading. Journal of Portfolio Management, 21(3), 10-18. http://dx.doi.org/10.3905/jpm.1995.409518

Engle, R.F. (1982). Autoregressive conditional heteroscedasticity with estimates of the variance of U.K. inflation. Econometrica, 50(4), 987-1008.

Engle, R.F., \& Ng, V.K. (1993). Measuring and testing the impact of news on volatility. The Journal of Finance, 48(5), 1749-1778. http://dx.doi.org/10.1111/j.1540-6261.1993.tb05127.x

Eom, K.S., Ok, J., \& Park, J.H. (2007). Pre-trade transparency and market quality. Journal of Financial Markets, 10(4), 319-341. http://dx.doi.org/10.1016/j.finmar.2007.06.001

Frutos, M.A., \& Manzano, C. (2014). Market transparency, market quality, and sunshine trading. Journal of Financial Markets, 17, 174-198. http://dx.doi.org/10.1016/j.finmar.2013.06.001

Heutschel, L. (1991). The absolute value GARCH model and the volatility of U.S. stock returns. Unpublished Manuscript, Princeton University.

Kandel, E., Rindi, B., \& Bosetti, L. (2012). The effect of a closing call auction on market quality and trading strategies. Journal of Financial Intermediation, 21(1), 23-49. http://dx.doi.org/10.1016/j.jfi.2011.03.002

Madhavan, A. (1992). Trading mechanisms in securities markets. Journal of Finance, 47(2), 607-641. http://dx.doi.org/10.1111/j.1540-6261.1992.tb04403.x

Madhavan, A., \& Panchapagesan, V. (2000). Price discovery in auction markets: A look inside the black box. The Review of Financial Studies, 13(3), 627-658. http://dx.doi.org/10.1093/rfs/13.3.627

Madhavan, A., Porter, D., \& Weaver, D. (2005). Should securities markets be transparent? Journal of Financial Markets, 8(3), 266-288. http://dx.doi.org/10.1016/j.finmar.2005.05.001

Pagano, M.S., Peng, L., \& Schwartz, R.A. (2013). A call auction's impact on price formation and order routing: Evidence from the NASDAQ Stock Market. Journal of Financial Markets, 16(2), 331-361. 
http://dx.doi.org/10.1016/j.finmar.2012.11.001

Pinfold, J.F., \& He, D. (2012). The impact of introducing a pre-close on the New Zealand Share Market. Journal of Financial Regulation and Compliance, 20(1), 99-110. http://dx.doi.org/10.1108/13581981211199443

Reboredo, J.C. (2012). The switch from continuous to call auction trading in response to a large intraday price movement. Applied Economics, 44(8), 945-967. http://dx.doi.org/10.1080/00036846.2010.526584

Ronen, T. (1998). Trading structure and overnight information: a natural experiment from the Tel-Aviv Stock Exchange. Journal of Banking and Finance, 22(5), 489-512. http://dx.doi.org/10.1016/S0378-4266(98)00024-7

Schwartz, R.A. (2000). Building a better stock market: new solutions to old problems. Working Paper, AEI-Brookings Joint Center For Regulatory Studies.

Shah, A., \& Sivakumar, S. (2000). Changing liquidity in the Indian equity market. Emerging Markets Quarterly, 4(2), 62-71.

Zhang, J., \& Hu, W. (2013). Does realised volatility provide additional information? International Journal of Managerial Finance, 9(1), 70-87. http://dx.doi.org/10.1108/17439131311298539

\section{Notes}

Note 1. Observations pertaining to particular days when one of the exchanges was closed for trading were deleted from the sample.

Note 2. Tests for volatility asymmetry indicated that it was not necessary to account for this feature. We used an asymmetric volatility test where the squared error term from an AR(1) model was regressed over an intercept, an error term and a dummy variable of the lagged error sign which denotes asymmetric volatility (Engle and $\mathrm{Ng}$, 1993). The dummy variable was insignificant, with a coefficient of 0.00009 and a t-ratio of 1.09 . 